UDC 517.977, 519.715

A. N. KiRILLOV

\title{
ON THE STABILIZATION OF THE LINEAR HYBRID SYSTEM STRUCTURE
}

\begin{abstract}
The linear control hybrid system, consisting of a finite set of subsystems (modes) having different dimensions, is considered. The moments of reset time are determined by some complementary function - evolutionary time. This function satisfies the special complementary ordinary differential equation. The mode stabilization problem is solved for some class of piecewise linear controls. The method of stabilization relies on the set of invariant planes, the existence of which is due to the special form of the hybrid system.
\end{abstract}

Key words: stabilization, variable structure, hybrid system 2010 Mathematical Subject Classification: 93B12

1. Introduction. The variable structure linear dynamical systems, i.e. the systems changing, while functioning, their dimensions and coefficient matrices, belong to the class of hybrid systems. The hybrid systems are dynamical systems demonstrating interacting of continuous and discrete dynamics [1], [2]. Their applications involve mathematical modeling in robotics, biochemistry, chemical technology, air and ground transportation and so on.

Roughly speaking, the main components of these systems are the partition of the phase space into a finite set of subspaces, the set of subsystems (modes) with continuous dynamics (each subsystem is defined in its subspace), and the set of mappings realizing, in some sense, a transfer from one subspace to another. The moments of reset time may be determined, as it was proposed in [3], [4], by the solutions of complementary system of differential equations. In this paper the problem of stabilizing of modes is solved for the linear control hybrid system. The method of stabilization is developed. Otherwise speaking, this method permits to achieve and then to preserve the desired structure of the system.

(C) Petrozavodsk State University, 2014 
2. The controllable evolutionary time. Let us consider the hybrid linear system which consists of a set of linear subsystems, or modes, $S_{k}$

$$
\dot{X}_{k}=A_{k} X_{k},
$$

which dimensions $k$ depend on time, $k=k(t), k=1, \ldots, n$, and the coefficient matrices are piecewise constant. Suppose that a function $y=y(t)$, evolutionary time (in our terminology), satisfying the following conditions

$$
\begin{gathered}
\dot{y}=B_{k}^{T} X_{k}+u, \\
y(t) \in \Delta_{k}=\left[y_{k}, y_{k+1}\right],
\end{gathered}
$$

is responsible for a changing of modes: the mode undergoes a changing when $y(t)$ becomes equal to $y_{k}$ or $y_{k+1}$ (the procedure of mode changing will be described below). Here $\mathbb{R}^{k} \supset X_{k}^{T}=\left(x_{1}, \ldots, x_{k}\right)$ - a system state, $T$ - the symbol of transposition, $\mathbb{R} \ni y(t)$ - an evolutionary time, $A_{k}=$ $=\left\{a_{i j}\right\}-$ a square constant matrix of order $k, B_{k}^{T}=\left(b_{1}, \ldots, b_{k}\right) \in \mathbb{R}^{k}-$ a constant vector, $u$ - a control, $y_{k}$ - some given constants (thresholds), $y_{k}<y_{k+1}, y_{1}=-\infty, y_{n+1}=+\infty$. We say that the system $S$, described by equations (1)-(3), is in the state $S_{k}$.

It is essential that the system (1), (2) has invariant sets [5], namely the integral planes

$$
\Pi_{k}=\left\{\left(x_{1}, \ldots, x_{k}, y\right): \alpha_{1} x_{1}+\ldots+\alpha_{k} x_{k}+\beta_{k} y=c\right\}
$$

with constant $c$, where $\left(\alpha_{1}, \ldots, \alpha_{k}, \beta_{k}\right)$ is a nonzero solution of the system

$$
a_{1 i} \alpha_{1}+\ldots+a_{k i} \alpha_{k}+\left(b_{i}+p_{i}\right) \beta_{k}=0, \quad i=1, \ldots, k .
$$

The procedure of mode changing: when the trajectory of the system (1), (2) attains the plane $y=y_{k}$ at some moment of time $t_{k}$, the transfer from the state $S_{k}$ to the state $S_{k-1}$ occurs, while hitting the plane $y=$ $=y_{k+1}$ at some moment of time $t_{k+1}$ means the transfer from $S_{k}$ to $S_{k+1}$. The transfer maps $\varphi_{k, k-1}\left(\varphi_{k, k+1}\right)$ from $S_{k}$ to $S_{k-1}$ (from $S_{k}$ to $S_{k+1}$ ) have the following form

$$
\varphi_{k, k-1}: Z_{k} \rightarrow C(k-1, k) Z_{k}+E_{k-1}(-\varepsilon),
$$

where $Z_{k}=\left(x_{1}, \ldots, x_{k}, y_{k}\right)^{T}, E_{k-1}(-\varepsilon)=(0, \ldots, 0,-\varepsilon)^{T},(-\varepsilon)$ is at $k$-th place, with

$$
0 \leq \varepsilon<\min _{k}\left(y_{k+1}-y_{k}\right), k=1, \ldots, n-1,
$$


$C(k, k-1)$ - the $k \times(k+1)$ constant matrix with elements $c_{i j}$, where

$$
\begin{gathered}
c_{k, k+1}=1, c_{k, j}=0, j=1, \ldots, k, c_{i, k+1}=0, i=1, \ldots, k-1, \\
\varphi_{k, k+1}: Z_{k} \rightarrow D(k, k+1) Z_{k}+E_{k+2}(\varepsilon),
\end{gathered}
$$

where $D(k, k+1)$ - the $(k+2) \times(k+1)$ constant matrix with elements $d_{i j}, d_{k+2, k+1}=1, d_{k+2, j}=0, j=1, \ldots, k, d_{i, k+1}=0, i=1, \ldots, k+1$. We suppose that $\varphi_{k, k-1}\left(Z_{k}\right),\left(\varphi_{k, k+1}\left(Z_{k}\right)\right)$ are the initial data for the states $S_{k}\left(S_{k+1}\right)$ and the switching occurs instantaneously.

Remark 1. The transfer matrices $C(k, k-1), D(k, k+1)$ are supposed to be constant. It is not essential for the obtained results which remain valid for the elements of $C(k, k-1), D(k, k+1)$ dependent on time or $k$.

Let us formulate the problem: we need to construct a control $u$ which transfers the system $S$ from a state $S_{k}$ to a state $S_{m}, \quad k, m \in\{1, \ldots, n\}$, $k \neq m$, in a finite time and then preserves the terminal state $S_{m}$.

Assume that the admissible control is $u=p_{1} x_{1}+\ldots+p_{k(t)} x_{k(t)}=$ $=P_{k}^{T} X_{k}$, where $p_{i}=p_{i}(t)$ are the piecewise constant coefficients. Here $k(t)$ is the integer valued function: $k(t) \in\{1, \ldots, n\}$. Thus $u$ belongs to a class of the piecewise linear functions of $X_{k(t)}$.

Remark 2. This problem differs from the traditional controllability or stabilizability problems. The proposed system is described by a continuous state $\left(X_{k}, y\right)$ as well as by a discrete state $k$, and we control the latter. It may be said that a discrete state represents the structure of the system. Thus it is apposite to designate the problem formulated above as the problem of the structure stabilization.

Below we propose the method of the structure stabilization. Assume that for $k=1, \ldots, n$ the systems (1) are asymptotically stable. The method consists of three steps. Consider the transfer $S_{k} \rightarrow S_{k+1}$.

1. Let $t_{k}\left(X_{k 0}, \delta\right)$ be such moment of time that $\left\|X\left(t, X_{k 0}\right)\right\| \leq \delta$ $\left(\|\cdot\|\right.$ is an euclidean norm) for $t \geq t_{k}\left(X_{k 0}, \delta\right)$ and some $\delta>0$, where $X\left(t, X_{k 0}\right)$ is the trajectory of the system (1), satisfying the initial condition $X\left(0, X_{k 0}\right)=X_{k 0}$. It is clear that $t_{k}\left(X_{k 0}, \delta\right)$ exists due to the asymptotic stability of (1). Let us take $p_{i}=-b_{i}, i=1, \ldots, k$, while $t \leq t_{k}\left(X_{k 0}, \delta\right)$. As a result the trajectory, remaining in $\mathbb{R}^{k} \times \Delta_{k}$, gets into the cylinder

$$
U_{\delta}(0)=\left\{X_{k} \in \mathbb{R}^{k}:\left\|X_{k}\right\| \leq \delta\right\}
$$

and remains there. 
2. At the moment $t=t_{k}\left(X_{k 0}, \delta\right)$ we take

$$
u=p_{1} x_{1}+\ldots+p_{k(t)} x_{k(t)}=P_{k}^{T} X_{k},
$$

where $p_{i}, i=1 \ldots, k$, satisfy the following inequality

$$
\begin{gathered}
\sum_{j=1}^{k} p_{j} \cdot \sum_{i=1}^{k} \hat{A}_{(k-1), i}(j) x_{i 0}> \\
>\operatorname{det} A_{k}\left(y_{k+1}-y_{0}\right)-\sum_{j=1}^{k} b_{j} \cdot \sum_{i=1}^{k} \hat{A}_{(k-1), i}(j) x_{i 0},
\end{gathered}
$$

if $\operatorname{det} A_{k}>0$ (for $\operatorname{det} A_{k}<0$ the sign of inequality is opposite), where $\hat{A}_{(k-1), i}(j)$ is a cofactor of the $j$-th row and the $i$-th column elements of $A_{k i}$ - the matrix obtained from $A_{k}$ via substituting of it's $i$-th column by $-\left(b_{1}+p_{1}, \ldots, b_{k}+p_{k}\right)^{T}$. The existence of the inequality solution was proved in [6].

The main idea of this step is that the vector $\left(p_{1}, \ldots, p_{k}\right)$, satisfying inequality, given above, provides such position of the integral plane $\Pi_{k} \subset$ $\subset \mathbb{R}^{k} \times \mathbb{R}$, for which $\Pi_{k}$ intersects the $Y$ axis with $y>y_{k+1}$ [6]. Then, in view of asymptotical stability of the equilibrium of the system (1) and taking into account that $\left\|X\left(t, X_{k 0}\right)\right\| \leq \delta$ for $t \geq t_{k}\left(X_{k 0}, \delta\right)$, we obtain that the trajectory intersects the plane $y=y_{k+1}$ in a finite time. As a result we obtain the transition to the state $S_{k+1}$.

3. Then let us put $p_{i}=-b_{i}, i=1, \ldots, k+1$, at the moment of time when $y=y_{k+1}+\varepsilon$, and the system preserves its state $S_{k+1}$.

Using the presented procedure, providing the transfer $S_{k} \rightarrow S_{k+1}$, we can obtain the transfer from any state $S_{k}$ to any state $S_{m}, \quad k, m \in$ $\in\{1, \ldots, n\}, k \neq m$.

The method of structure stabilization essentially relies on the asymptotic stability of the system (1). It means that each matrix $A_{k}$ has all eigenvalues with negative real parts. Let us consider the general hyperbolic case, e.g. the spectrum of $A_{k}$ has not common points with the imaginary axis. It is known that in this case we have the direct decomposition $\mathbb{R}^{k}=E^{s} \oplus E^{u}$, where $E^{s}, E^{u}$ are such invariant subspaces of the operator $L_{k}$, corresponding to $A_{k}$, that the eigenvalues of the restrictions $L_{k} \mid E^{s}$ have negative real parts and the eigenvalues of $L_{k} \mid E^{u}$ have positive real parts. If $X_{k 0} \in E^{s}$ then the method of structure stabilization remains the same, as it was presented above. 
Therefore we consider the case $X_{k 0} \notin E^{s}$. Then there exists such component $x_{j}(t)$ of $X\left(t, X_{k 0}\right)$ that $x_{j}(t) \rightarrow \infty$ as $t \rightarrow+\infty$. Thus we can take $p_{j}$ in the following form: $p_{j}=-b_{j}+c_{j}$ sign $x_{j}$, where $c_{j}>0-$ some constant, while $p_{i}=-b_{i}$ for $i=1, \ldots, k, i \neq j$. As a result (2) implies that the trajectory attains the plane $y=y_{k+1}$ in a finite time. Then, similarly to the third step of the method presented above, we put $p_{i}=-b_{i}, i=1, \ldots, k+1$. Thus we obtain the transfer $S_{k} \rightarrow S_{k+1}$.

3. The controllable continuous state. Now instead of (1), (2) we consider the controllable system

$$
\dot{X}_{k}=A_{k} X_{k}+G_{k} v_{k}, \quad \dot{y}=B_{k}^{T} X_{k},
$$

together with (3), where $v_{k} \in \mathbb{R}^{l}-$ a control, $G_{k}$ is a constant matrix. Then we can use the classical method of linear stabilization [7] in order to make the origin $X_{k}=0$ asymptotically stable. It is sufficient to find such matrix $M_{k}$ that the system $\dot{X}_{k}=\left(A_{k}+G_{k} M_{k}\right) X_{k}=N_{k} X_{k}$ be asymptotically stable, where

$$
v_{k}=M_{k} X_{k}
$$

Without loss of generality, but keeping in view the method of structural stabilization, we consider the case for which $v_{k} \in \mathbb{R}, G_{k} \in \mathbb{R}^{k}, M_{k} \in \mathbb{R}^{k}$.

If $\alpha_{1} x_{1}+\ldots+\alpha_{k} x_{k}+\beta_{k} y=\alpha_{1} x_{10}+\ldots+\alpha_{k} x_{k 0}+\beta_{k} y_{0}$ is the integral plane of the system

$$
\dot{X}_{k}=N_{k} X_{k}, \quad \dot{y}=B_{k}^{T} X_{k},
$$

then, analogously to [6], it is easy to show that $\alpha^{T}=\left(\alpha_{1}, \ldots, \alpha_{k}\right)$ satisfies the linear system

$$
N_{k}^{T} \alpha=-\beta_{k} B_{k},
$$

where $N_{k}=A_{k}+G_{k} M_{k}^{T}$. Let $\bar{y}$ be the coordinate of the intersection point of an integral plane with the axis $Y$ and assume that

$$
\bar{y}=\frac{1}{\beta_{k}} \sum_{i-1}^{k} \alpha_{i} x_{i 0}>y_{k+1} .
$$

It is well known, that if

$$
\operatorname{rank} L_{k}=\operatorname{rank}\left(G_{k} A_{k} G_{k} A_{k}^{2} G_{k} \ldots A_{k}^{k-1} G_{k}\right)=k,
$$


then we can find such $M_{k}$ that the matrix $N_{k}$ has any desired eigenvalues [7]. Let all eigenvalues be with the negative real parts, which provides the asymptotic stability of the system $\dot{X}_{k}=N_{k} X_{k}$. In addition [7]

$$
M_{k}=d^{T} P_{k}^{-1} L_{k}^{-1},
$$

where $P_{k}=\left(I_{k} p+e_{k}, I_{k}^{2} p+e_{k-1}, \ldots, I_{k}^{k} p+e_{1}\right), p=-L_{k}^{-1} A_{k}^{k} G_{k}, I_{k}=$ $=\left(\overline{0}, e_{1}, \ldots, e_{k-1}\right), \overline{0} \in \mathbb{R}^{k}$ is the zero vector, $e_{i} \in \mathbb{R}^{k}$ - the vector with zero components except the $i$-th component which equals 1 . The components $d_{i}$ of $d \in \mathbb{R}^{k}$ equal $d_{i}=p_{i}-q_{i}, i=0,1, \ldots, k-1$, where $p_{i}$ - the components of $p, q_{i}$ - the coefficients of the polynomial $Q_{k}(\lambda)$ which roots equal to the eigenvalues of the matrix $N_{k}$.

The result, presented below, provides only local stabilization. Assume that $X_{k 0} \in U_{\delta}(0)$, where $U_{\delta}(0)$ is the cylinder from section 2. Then for $v_{k}$, constructed above, the trajectory $X\left(t, X_{k 0}\right)$ remains in $U_{\delta}(0)$. The previous arguments imply the following theorem.

Theorem 1. Let $X_{k 0} \in U_{\delta}(0), X_{k 0} \neq 0$ and assumptions (7)-(10) are valid. Then the control $v_{k}=M_{k} X_{k}$, stabilizing the system $\dot{X}_{k}=A_{k} X_{k}+$ $+G_{k} v_{k}$, provides the transfer of the system (6), (3) from $S_{k}$ to $S_{k+1}$.

Remark 3. It is naturally to formulate the following problem: for which maximal set of $X_{k 0}$ the presented theorem is true?

4. Conclusion. The method of the linear hybrid system structure stabilization is proposed in this paper. The feedback piecewise linear control of the discrete state is constructed.

The hybrid systems, considered in this paper, have different applications in the problems of mathematical modeling, particularly in the economic dynamics. Thus, in [8] the linear model of the economic growth dynamics with the structural variations was proposed. The control growth problem was solved for the industrial group, consisting of several enterprises, the number of which was not constant, but depended on the economic efficiency and changed in time.

Future investigations will be dedicated to some extensions of the proposed methods of the structure stabilization, especially the constraints on the admissible controls and phase variables, specific for applications, will be taken into account.

Acknowledgment. This work was supported by the Programm of strategic development of the PetrSU. 


\section{References}

[1] Arjan van der Schaft, Hans Schumacher. An Introduction to hybrid dynamical systems. London, Springer, 2000, 174 p.

[2] Handbook of hybrid systems control. Theory, tools, applications. Ed. by J. Lunze. Cambridge University Press., 2009, 565 p.

[3] Roger W. Brockett. Hybrid models for motion control systems. Essays in Control: Perspectives in the Theory and its Applications. Ed. by H. L. Trentelman and J. C. Willems, Boston, Birkhauser, 1993, pp. $29-53$.

[4] Kirillov A. N. The systems with variable fhase space in modeling of biological wastewater treatment processes. The Russian conference "Mathematical problems of ecology". The conference abstracts. Dushanbe, 1991, p. 44.

[5] Kirillov A. N. The control of multistage technological processes. Vestnik Sankt-Peterburgskogo Universiteta. Seriya 10. Prikladnaya Matematika. Informatika. Protsessy Upravleniya, 2006, vol. 4, pp. 127-131.

[6] Galakhova M. E., Kirillov A. N. The linear variable structure system control. Proceedings of the Karelian Research Centre of the RAS. No.5. Mathematical modeling and information technologies, 2012, vol. 3, pp. 18-21.

[7] Smirnov E. Y. The stabilization of programmed movements. SaintPetersburg, SPbSU, 1997, 308 p.

[8] Kirillov A. N. The method of dynamical decomposition in modeling of variable structure systems. Information and Control Systems, 2009, no. 1 , pp. 20-24.

Received August 20, 2014.

In revised form, October 15, 2014.

Institute of Applied Mathematical Research

Karelian Research Centre, Russian Academy of Sciences

11, Pushkinskaya st., 185910 Petrozavodsk, Russia;

Petrozavodsk State University

33, Lenina st., 185910 Petrozavodsk, Russia

E-mail: kirillov@krc.karelia.ru 